Received: 02 July 2018

Accepted: 27 September 2018

Published online: 31 October 2018

\section{Metamaterial Superlenses Operating at Visible Wavelength for Imaging Applications}

\author{
S. Haxha ${ }^{1}$, F. AbdelMalek' ${ }^{2}$, F. Ouerghi' ${ }^{2}$, M. D. B. Charlton ${ }^{3}$, A. Aggoun ${ }^{4} \&$ X. Fang ${ }^{3}$
}

In this paper, a novel design for a metamaterial lens (superlens) based on a Photonic Crystal (PC) operating at visible wavelengths is reported. The proposed superlens consist of a gallium phosphide (GaP) dielectric slab waveguide with a hexagonal array of silver rods embedded within the GaP dielectric. In-house 2DFDTD numerical method is used to design and optimize the proposed superlens. Several superlenses are designed and integrated within a same dielectric platform, promoting the proof-of-concept (POC) of possible construction of an array of superlenses (or sub-lenses to create an M-Lens) for light field imaging applications. It is shown that the concavity of the superlens and positioning of each sub-lens within the array strongly affects the performances of the image in terms of resolution. Defects and various geometrical shapes are introduced to construct and optimize the proposed superlenses and increase the quality of the image resolution. It is shown that the orientation of the active region (ellipse) along $x$ and $y$ axis has tremendous influence on the quality of image resolution. In order to investigate the performance characteristics of the superlenses, transmitted power is calculated using 2D FDTD for image projections at various distances (in $x$ and $y$ plane). It is also shown, how the proposed superlens structures could be fabricated using standard micro fabrication techniques such as electron beam lithography, inductively coupled Reactive ion etching, and glancing angle evaporation methods. To the best of our knowledge, these are the first reported POC of superlenses, integrated in a monolithic platform suitable for high imaging resolution that can be used for light field imaging applications at visible wavelength. The proposed superlenses (integrated in a single platform M-Lens) will have tremendous impact on imaging applications.

Emerging superlenses with aberration correction have generated great attention in the field of the optical microscopy and imaging applications due to their ability to significantly increase the image resolution quality. These superlenses provide super image resolution by overcoming the classical diffraction limits of light propagation ${ }^{1-3}$. Although many lens imaging devices and systems have been realized, the negative refraction mechanism offered by Photonic Crystals (PC) devices enable high quality resolution at nanoscale ${ }^{4}$. Surface plasmon-polaritons based on PC structures offer a unique imaging lens approaches such as hyperlenses ${ }^{5-9}$ and/or metamaterial superlenses ${ }^{10,11}$. Advantages in understanding of the fundamental mechanisms of the light propagation has enabled a rapid progress in developing of devices and systems that offer high quality image resolution. These advanced light propagation control and manipulation approaches have provided unprecedented high quality resolution images.

PC structures, and metamaterial lenses based on PC structures for light coupling, light manipulations, photonic integration, biosensing and various imaging applications have been extensively reported in the literature $^{12-20}$. Modulating dispersion properties of PC based lens with low index and tunable optical properties using various microfluidics and as used as an optical coupling element operating a various wavelengths $(0.75-3 \mu \mathrm{m})$ have been discussed $\mathrm{in}^{12}$. Spatially-variant PC structures for integrated photonics, where the alignment of the unit cell varies as a function of position capable of sharply guiding light beams using just low index materials with very high polarization selectivity is reported in ${ }^{13}$. In this ref. ${ }^{13}$, a PC structure configuration for controlling the

${ }^{1}$ Royal Holloway, University of London, Microwave Photonics and Sensors group Department of Electronic Engineering, Egham, Surrey, TW20 0EX, United Kingdom. ${ }^{2}$ Advanced Materials and Quantum Phenomena Laboratory, Faculty of Science at Tunis, University of El Manar, Tunis, Tunisia. ${ }^{3}$ Electronics and Computer Science, University of Southampton University Road Southampton, SO17 1BJ, Southampton, United Kingdom. ${ }^{4}$ School of Mathematics and Computer Science, University of Wolverhampton Wulfruna Street Wolverhampton, WV1 1LY, Wolverhampton, United Kingdom. S. Haxha, F. AbdelMalek and F. Ouerghi contributed equally. Correspondence and requests for materials should be addressed to S.H. (email: Shyqyri.Haxha@rhul.ac.uk) 
light is proposed where the infrared light of one polarization is propagating through sharp bends, whereas the other polarization of the light is propagating straight through the spatial variant PC. PC lens based on ultra-sharp nano-focusing of graded index with optimized single defect and with the beam spot size (Full width at half maximum $(\mathrm{FWHM})=\lambda / 75)$ that could be used for fluorescent imaging analysis, biosensing and sub-surface nano-metrology applications are reported $i^{14}$. A PC lens structure with high coupling efficiency is reported in ref. ${ }^{15}$, despite differences between the theoretical and experimental obtained results, the reported PC lens ${ }^{15}$ can still be used as an effective method for light coupling in $\mathrm{THz}$ integrated circuits. A PC planar lens operating at microwave (low) frequencies using the ray tracing method has been reported in ${ }^{16}$. Various designs, using FEM-based numerical simulations, of plano-concave lenses with epsilon-near-zero surface-relief coatings for efficient shaping of nonparaxial optical beams are reported in ${ }^{17}$. Plano-concave silicon lenses based on coupled metacoatings set at the entrance and exit surfaces of a transparent dielectric thick lens structure with a potential applications in optical trapping and detection is reported in ${ }^{18} \cdot \operatorname{In}^{19}$ two types of binary $2 \mathrm{D}$ subwavelength (operating at wavelength was $\lambda=10 \mathrm{~mm}$ ) focus diffractive PC lens is reported. Numerous PC types of structural disorders in the hole diameter and to interface roughness, including the imperfection disorder in the lattice periodicity in terahertz time-domain spectroscopy are reported $\mathrm{in}^{20}$. It is reported that guided resonances in the PC lens structures are prone to any potential hole diameter disorders and interface roughness, nevertheless they are more sensitive to lattice periodicity fabrication imperfections. It should also be noted that, in our previous work reported in ${ }^{21}$, we have reported a planoconcave metamaterial lens based on PC structure for integration with a Single Mode Fiber (SMF) and an ordinary photonic crystal waveguide.

In this study, we have proposed a novel lens with unique structure based on a combination of metamaterials and engineering of PC structure geometry shape, defects ${ }^{11}$, and introduction of reflectors are included. By altering and optimising various lens structure parameters, the ray focus position at any desired location would have significant contributions to light field imaging applications. Light field imaging technology was introduced by Lippman in $1908^{22}$. Light field imaging emulates the visual system of insects, specifically the fly, where micro-lenses are used to capture an object section or a part, enabling $3 \mathrm{D}$ content imaging by using a single camera snapshot. This technique offers improved imaging while enhancing the 3D view without any additional visual assistance. Light field has been attracting a significant amount of interest in recent year due to its property to provide refocus after shoot, depth computation and 3D replay ${ }^{23-25}$. An important observation from the Lipman concept is that the replayed image captured through an micro-lens array will result in an inverted depth ${ }^{26}$. Previous solutions use a double micro-lens array to invert the image before recording which makes the capture system cumbersome ${ }^{26}$. Metamaterials are new types of materials which refract light in the opposite direction to conventional materials. Hence, a negative refractive index metamaterial refracts the light on the same side of the normal as the incident beam, rather than on the opposite side as in conventional materials. Using a micro-lens array where each micro-lens is made of negative refractive index metamaterial lens will resolve this problem. Example of Raytrix Light field imaging system uses micro-lens arrays with various micro lens forms which vary in their focal length to allow extension of the depth of field. Using conventional micro-lens structure will make the fabrication process complex. Using Metamaterials allows several similar/identical or different superlens platforms can be designed depending on the applications and hence offers greater opportunities to extend the depth of field for greater depth computation.

Using Metamaterials allows several similar/identical or different superlens platforms can be designed depending on the applications and hence offers greater opportunities to extend the depth of field for greater depth computation. Current conventional microlens arrays suffer from high losses, low (poor) resolution, inability to determine the depth of an object in the third dimension, and have limited angle of view $(\mathrm{AOV})^{27-31}$. It is also known that the resolution of current optical devices is limited by the wavelength of light. In particular, conventional lenses are unable to image objects smaller than half the illumination wavelength. The proposed superlens would have significant advantages when capturing an object image, and can be designed to fit easily within a conventional pixel size. Therefore our proposed metamaterial superlenses will have great impact on enhancing the resolution and projection of light field imaging system. The proposed lens based on metamaterial PC structure would provide significant enhanced image processing functionality. By engineering the geometry and optimizing the design parameters of the PC structures, the focus of the light can be tuned to the surface of the lens where the novel configuration of metasurface is able to overcome the light diffraction limits. Along the propagation of the light, the wavefront of the waves is constructed where the light can be used to generate a high imaging resolution. Since, the light propagates along the PC structure, its intensity decays, when it interacts with a sub-wavelength aperture, the intensity increases and the collection of the light wavelength can be performed. The proposed superlens will enable and simplify image transformations such as rotation, orientation, scaling and lighting changes. To the best of our knowledge, this is the first reported superlenses that can be integrated in an array suitable for high imaging resolution operating at visible wavelength. Designing and integrating an array of precise superlenses into a single lens platform will lead to a new class of imaging lens systems which will exhibit high resolution beyond existing state-of-the-art ${ }^{27}$.

\section{Simulation Method}

The proposed PC based superlens structures are simulated by using in-house 2D Finite Difference Time Domain (FDTD) method $^{32}$. In order to design, analyze and optimize the proposed PC superlens structure parameters, we have employed our in-house accurate 2D FDTD with a computational window enclosed by Perfectly Matched Layers (PML) ${ }^{33-37}$. The FDTD method ${ }^{33}$ is the most effective established numerical method for designing of metamaterial PC based devices. The FDTD method is considered a simple numerical method that enables modelling of complex periodic structures by solving directly using Maxwell's equation. The foundation of this method was proposed by Yee ${ }^{34}$. In ref. ${ }^{34}$ the author used differential and integral Maxwell's equations to represent the geometrical spatial sampling of the vector components of the magnetic and electric fields. The numerical technique 
introduced in ref. ${ }^{34}$ represents the FDTD time and space second order accuracy, where the numerical dispersion effects can be kept at a smaller size than $\lambda / 20$ or $\lambda / 30$. The stability of the FDTD algorithm in ref. ${ }^{34}$ requires an upper bound on the time step $(\Delta t)$ that is determined by the spatial increments $\Delta x, \Delta y$, and $\Delta z$ in accordance.

\section{Structure Fabrication Method}

The proposed PC superlens structure could be fabricated using standard micro fabrication techniques such as electron beam lithography, inductively coupled Reactive ion etching, and glancing angle evaporation methods. In practice the device would be implemented as a three layer slab waveguide providing a Planar Light Circuit (PLC) platform for device testing. This would consist of a thin $(400 \mathrm{~nm}) \mathrm{GaP}$ core layer grown epitaxially on a transparent Sapphire substrate, which provides a transparent underlying waveguide buffer layer. An overcoat top layer of $\mathrm{SiO} 2$ would be deposited by Plasma Enhanced Chemical Vapour Deposition (PECVD) or reactive sputtering, to form a top cladding layer. The pattern consisting of an array of holes would be defined using electron-beam lithography according to the above layout. The pattern would be transferred through the $\mathrm{SiO} 2$ to the GaP layer by inductive coupled reactive ion etching using $\mathrm{CHF} 3$ and $\mathrm{BCl} 3$ dry etch chemistry and a durable resist mask (ZEP). The Inductive coupled plasma process conditions can be adjusted to provide straight vertical side walls through the volume of the core layer (aspect ratios of 4:1 are easily achievable). With the remaining resist mask in place, the structure would then be coated with a thin silver layer by glancing angle e-gun evaporation. This ensures preferential coating of the inside walls of the holes as opposed to the top and bottom surfaces. Thickness is controlled to $\mathrm{nm}$ precision by a crystal monitoring system. For very thin conformal layers, electroplating could be used to grow the silver rings to the required thickness. Resist would then be removed by conventional metal lift off process to reveal a clean metal free top $\mathrm{SiO} 2$ surface.

Finally the entire structure would be over-coated with $\mathrm{GaP}$ material by reactive sputtering process to back-fill the central part of the holes. This will overcoat the top $\mathrm{SiO} 2$ surface as well, however this since layer is optically isolated from the $\mathrm{GaP}$ waveguide core by the top $\mathrm{SiO} 2$ layer, it does not interfere with optical transmission through the underlying $\mathrm{GaP}$ core layer. If necessary the residual $\mathrm{GaP}$ layer could be removed from the top surface by a planarization etch.

To couple light into the device grating couplers would be etched into the PLC to couple light into the device from the top. Scanning Near field Optical Microscopy (SNOM) would be used to test operation of the device. This involves positioning a tapered optical fiber over the top of the device, which in turn probes the evanescent field or scattered leakage light, and so provides a method to map out the optical field distribution in a practical way, for comparison to the field maps above.

\section{Dispersion in Ag Array}

It is already know that in PC structures that consist of metals, such as silver cylinders considered in this study, most electrons are free since they are not bound to the nucleus, therefore the restoring force is insignificant and there is no natural frequency occurring. Hence for the silver material dispersion, we have derived the Drude model by assuming;

$$
\varepsilon_{r}(\omega)=\varepsilon_{\infty}-\frac{\omega_{p}^{2}}{\omega^{2}+i \omega \Gamma}
$$

This equation (2) can be written in terms of the real and imaginary components.

$$
\varepsilon_{r}(\omega)=1-\left(\frac{\omega_{p}^{2} \tau^{2}}{1+\omega^{2} \tau^{2}}\right)+j\left(\frac{\omega_{p}^{2} \tau / \omega}{1+\omega^{2} \tau^{2}}\right)
$$

where the volume plasma frequency $\omega_{p}$ represents the effective mass of the electron $m *$ and its density $N$, hence $\omega_{p}=\left(N e^{2} / \varepsilon_{0} m *\right) 1 / 2$. The relaxation rate $\Gamma$ defines the effective electron scattering rate, with a matching relaxation time $\tau=1 / \Gamma, \varepsilon_{\infty}$ represents the net contribution from the positive ion cores. For the ideal free electron gas $\varepsilon_{\infty}=1$, and for metals $\varepsilon_{\infty}=1-10$ depending on the interband response ${ }^{38,39}$.

\section{Results and Discussions}

The proposed PC lens structure is shown in Fig. 1, which consists of a hexagonal PC lattice made of silver rods $(\mathrm{Ag})$ embedded in a dielectric background $(\mathrm{GaP})$ material. The dielectric constant, $\varepsilon_{\mathrm{GaP}}$ of the GaP is 12.25 and the radii of the Ag rods are equal to $r_{1}=0.2 a$ and $r_{2}=0.38 a$, where $a$ is the lattice constant and it is equal to $142 \mathrm{~nm}$. The operating wavelength is $\lambda_{0}=483 \mathrm{~nm}$ in the blue regime. In the right side of the PC structure, a concave region with radius $R$ is introduced as a defect. The dimensions of the structure in $x$ and $y$ direction are $16 \times 142 \mathrm{~nm}$ and $30 \times 142 \mathrm{~nm}$, respectively, and $R=5.3 a$. The source is located at $284 \mathrm{~nm}$ from the input of the PC metamaterial lens and the thickness of the simulation PML is fixed at 12 layers.

The dynamic process of the proposed PC structures are simulated with the following mesh sizes structure; $d x=d y=a / 30$, where $a$ is the lattice constant of the PC structure. In our calculations, we have used the Transverse Magnetic (TM) mode with the $\boldsymbol{E}_{z}, \boldsymbol{H}_{\boldsymbol{x}}$ and $\boldsymbol{H}_{\boldsymbol{y}}$ components. The incident light is a broad Gaussian source centered at the frequency $f=0.293 a / \lambda$. At the output of the PC structure a detector is placed to measure the light intensity. The transmission spectra along with the light intensity are determined at this frequency.

In this PC lens structure, the hexagonal PC lattice is selected based on the fact that they exhibit higher symmetry when compared to the PC rectangle lattice. The Brillouin zone is hexagone and its band gap is complete for both polarizations TE and TM. Hexagon PC lattice is larger than rectangle PC lattice. Also, the hexagonal PC lattice will be able to confine the light in $3^{\text {rd }}$ dimension when it is designed in $3 \mathrm{D}$. The selection of the inner and outer radius of Ag rod is based on the focusing of the emerging beam, the optimal dimensions are achieved when 
(a)

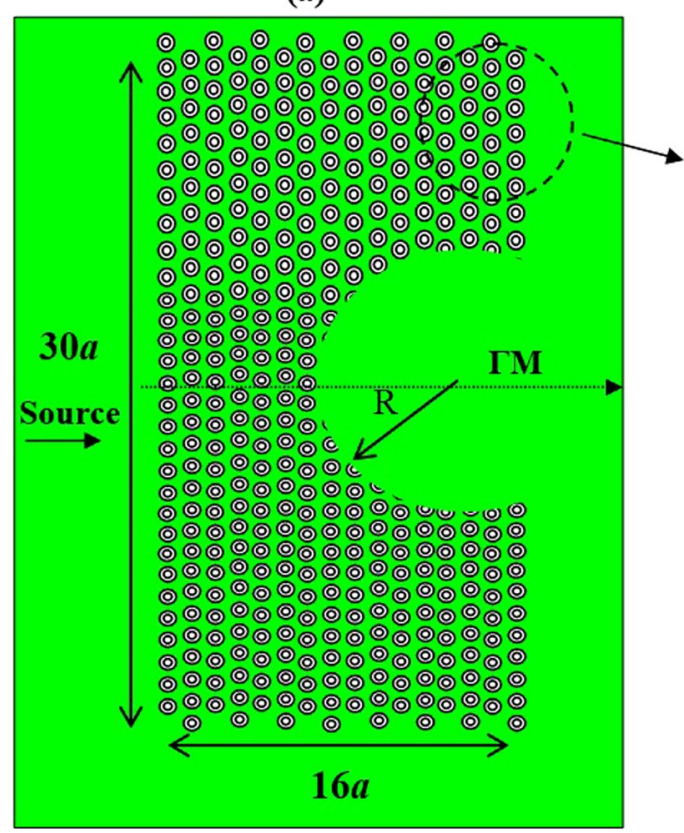

(b)

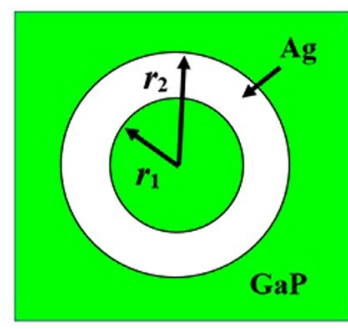

$$
\begin{aligned}
& r_{1}=0.2 a \\
& r_{2}=0.38 a \\
& \mathbf{R}=5.3 a
\end{aligned}
$$

Figure 1. Schematics of the proposed PC metamaterial lens: (a) structure geometry consisting of a hexagonal array of $\mathrm{GaP} / \mathrm{Ag} / \mathrm{GaP}$; (b) inset of the unit cell of the periodic structure. The inner radius $r_{1}$, outer radius $r_{2}$ and the radius $R$ of the concave region are defined in the image.

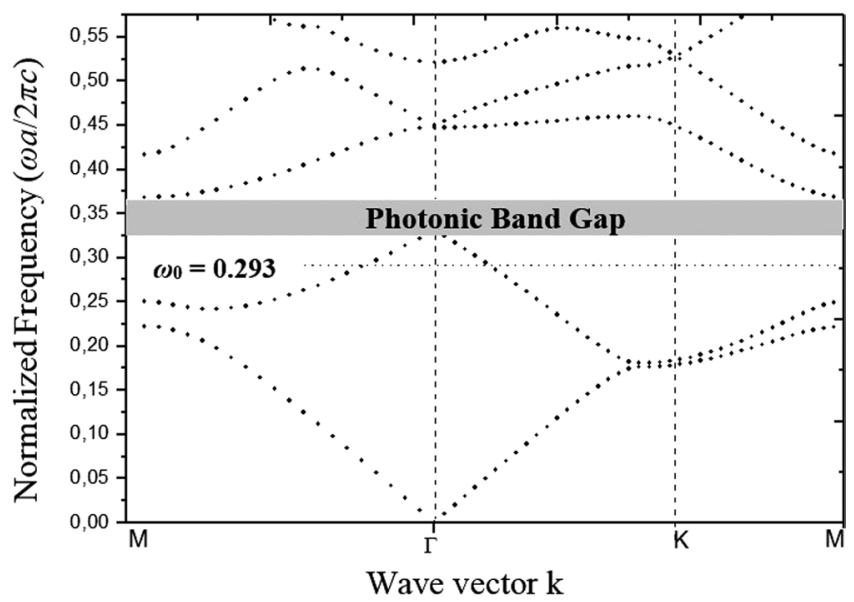

Figure 2. TM band diagram of the proposed metamaterial structure.

the image field is almost similar to the source. The selection of inner and outer radius of the Ag rod and the frequency band enables this structure to behave as metamaterial lens operating at visible wavelength.

The metamaterial lens light behaviour, illustrated in Fig. 2, is fundamentally different from the behaviour of a conventional fully dielectric 2D PC structure. The use of $\mathrm{GaP} / \mathrm{Ag} / \mathrm{GaP}$ materials, strongly modifies the light behaviour for the TM polarisation.

By analysing the photonic band diagram of the metamaterial lens, we found that the frequency region of the effective negative refraction exists in the second band in the visible domain with the following parameters; $\mu=0.293, \lambda_{0}=0.483 \mu \mathrm{m}$, as shown in Fig. 2 . In this case, the gradient of the bands indicates that the electromagnetic waves propagate with negative group velocity, and evanescent waves can be supported to perform high image resolution. In other words, significant image resolution is obtained at visible region by carefully designing and optimizing geometrical parameters of the structure shown in Fig. 1. It is worth stating that the quality and size of the formed image is depending on the following condition; $\left(\vec{V}_{\mathrm{g}} \cdot \overrightarrow{\mathrm{k}}\right)<0$ and $\left.\mathrm{V}_{\varphi} \cdot \mathrm{V}_{\mathrm{g}}\right)<0$ where $\vec{V}_{\mathrm{g}}$ is the group velocity, $\vec{k}$ is the wave vector and $V \varphi$ is the phase velocity. When constructing the structure geometry, initially as a first step, we remove silver rods to form $a$ in concave defect, and simulate the snap shot of the electric field. The obtained image of the source located on the left of the PC structure at distance $a$ is illustrated in Fig. 3. 


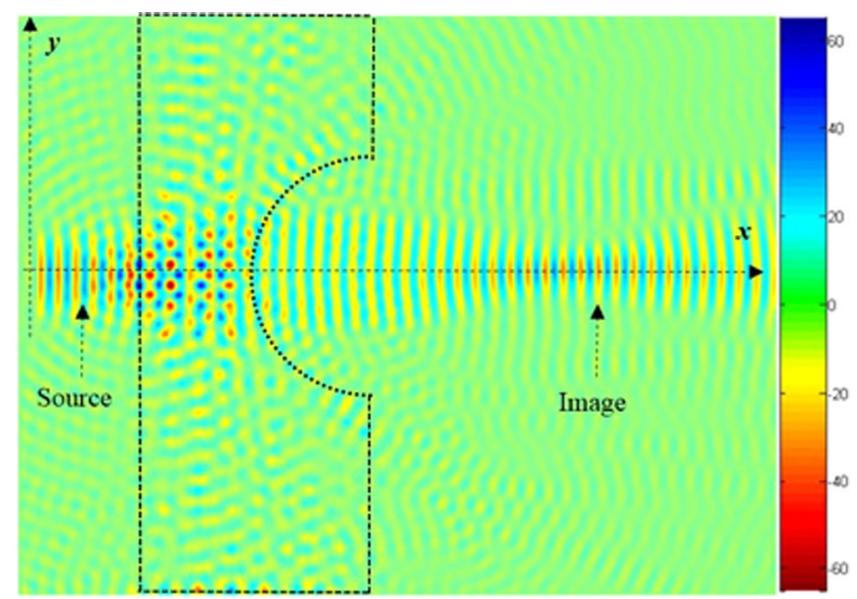

Figure 3. Superlens obtained image at $\lambda_{0}=0,483 \mu \mathrm{m}$, when the point source is $284 \mathrm{~nm}$ and a focus is obtained at $3,6 \mu \mathrm{m}$ from the source.

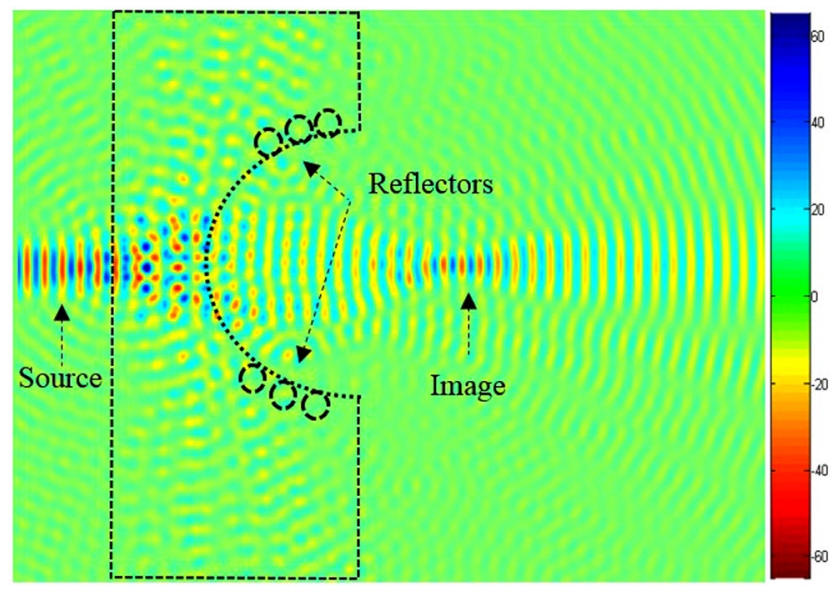

Figure 4. Reconstructed image of the transmitted field at $0,483 \mu \mathrm{m}$, with a radius $R^{\prime}=0.28 a$ of the reflector rods. Focusing is observed at $3,6 \mu \mathrm{m}$ away from the source. The reflectors shown in the inset picture are based on $\mathrm{GaP}$ rods.

In order to increase the resolution of the superlens, we have introduced reflectors on the concave region as illustrated in Fig. 4. The reflectors are rods with larger radius compared to the previous ones, which are considered as defects. While still keeping the source at the same distance $a$ from the PC structure, it is clear from this figure that the image is well confined when defect radius is $R^{\prime}=0.28 a$. Throughout this work $R^{\prime}$ is set to be equal to $0.28 a$.

The promise of perfect aberration free, ultra-high resolution optical imaging could revolutionize nanofabrication technology. To this end, we design a structure enabling perfect optical imaging where the structure is bounded by a reflector composed of GaP rods, as shown in Fig. 4. As can be seen from this figure, the image is clearly formed.

Figure 5 shows the transmission spectra of the light propagating through the PC lens. As can be seen in this spectrum, the amplitude of the transmission reaches $90 \%$ centered at $0.29 a / \lambda$ when the incident light is polarized parallel to rods (TM polarization). While in the spectral range $0.2 a / \lambda$ and $0.25 a / \lambda$, the amplitude is significantly decreased and drops to zero. The diffraction losses of quasi-guided modes are found to be complex functions of mode index and the wavevector. Losses depend on triangular lattice filling fraction, this strongly affects the quality of the image resolution. However, these diffraction losses have impact on the imaging process ${ }^{40}$.

To improve image quality (minimize losses), we introduce reflectors at the edges of the concave lens (shown in Fig. 4). Since in the absence of reflectors, the quality of the image is not clear, as illustrated in Fig. 6. The introduced reflectors contribute to reduce the losses of the light propagation, therefore the beam converges to the plane image which enhances the image quality. It should be stated that the curvature of the lens affects the number of the defects, and therefore influences the quality of the formed image. The introduction of reflectors converge the light to the image centre.

It is clear that enhancement of image resolution is possible if the superlens is concave, and it may be possible to use it as a magnifying superlens in terms of resolution and image location. Based on these properties, we 


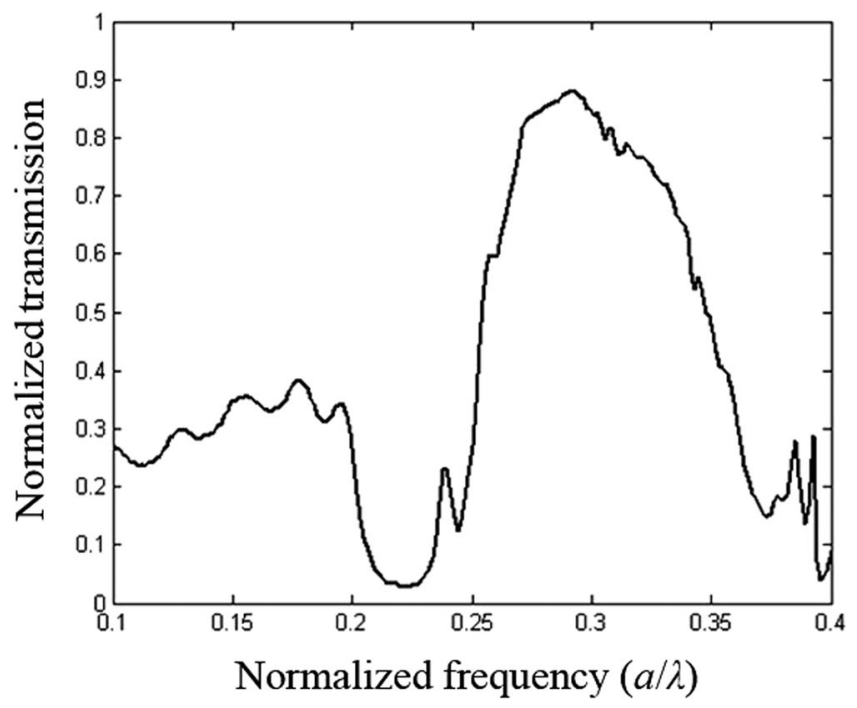

Figure 5. Transmission spectra centred at $\lambda_{0}=0.483 \mu \mathrm{m}$ for the TM polarization, including the upper and lower reflectors with a radius $R^{\prime}=0.28 a$. The propagation direction is $\Gamma$ K.

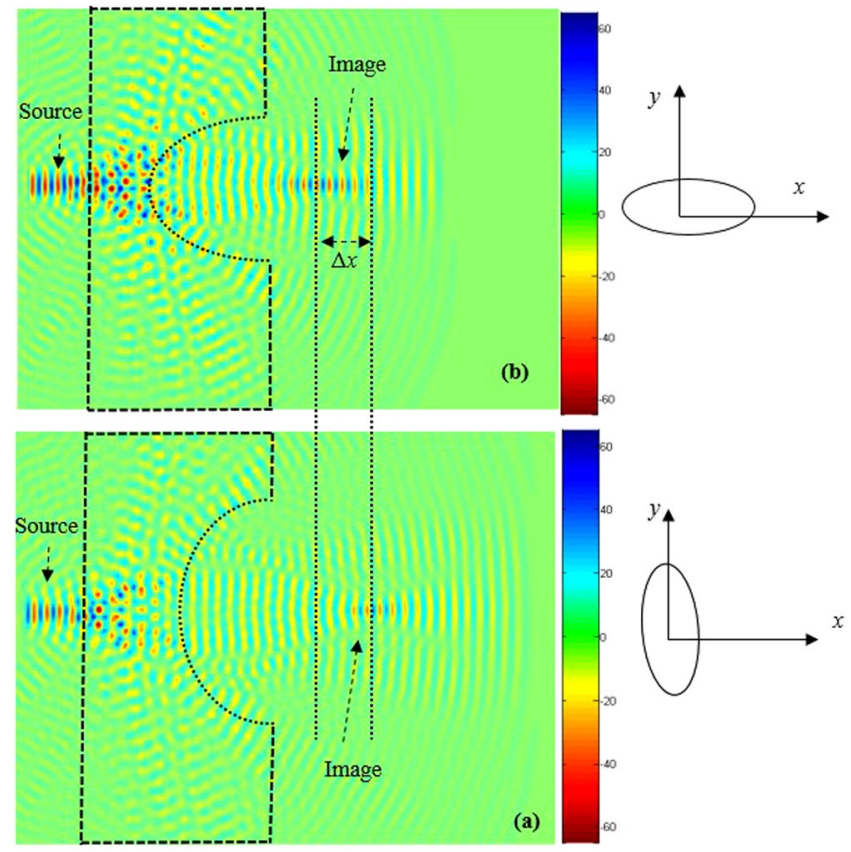

Figure 6. Source and the corresponding image formed by the superlens for a source at wavelength $\lambda_{0}=0.483 \mu \mathrm{m}$ when the concave region of the superlens is elliptical: (a) When the major axis of the elliptical defect is along the $x$-axis, (b) When the major axis of the elliptical defect is along the $y$-axis.

investigate the electromagnetic response of the PC structure when the concave region of the superlens is elliptical. We study the effect of the radius of the ellipse and its orientation on the displacement of the image, while keeping the fixed source at the distance $a=142 \mathrm{~nm}$. The following structure geometrical parameters are consider; the length of the minor axis of the ellipse along $x$-direction is $4 a$ and the length of the major axis along the $y$-direction is $5 a$. The electric field snapshot is illustrated in Fig. 6 . We can clearly notice that a sharp image of the object (the source) is formed. By reversing the orientation of the ellipse: i.e the major and the minor axis are along $x$ and $y$ direction, respectively, the image is shifted along $x$ - direction with a distance $\Delta x=142 \mathrm{~nm}$, as shown in Fig. 6b). Based on the dispersion relation, effective refractive indices for unit cells with differentrod radii are calculated.

In order to shift the image along $x$ - direction over long distance (far field), we optimize the dimensions of the ellipse (the minor axis of length $3 a$ is following the $x$-direction and the major axis length of $6 a$ according to the $y$ - direction). Figure 7 a shows the position of the image obtained with this configuration. By reversing the orientation of the ellipse, the image is strongly affected and shifts to a longer distance of about $\Delta x=2 a=284 \mathrm{~nm}$. 


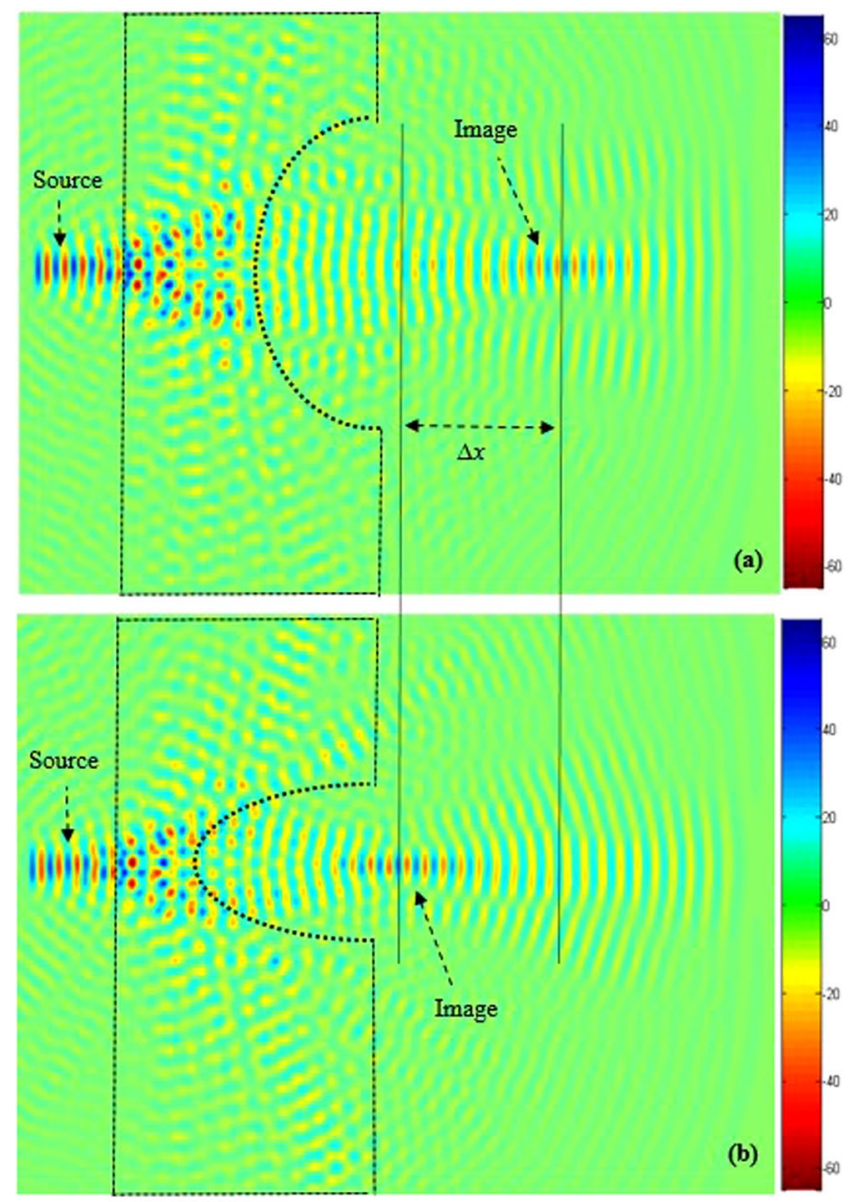

Figure 7. Time domain snapshots of the propagation beam when defects have been introduced in the PC superlens structure in order to realize a perfect imaging. Two different orientation of the elliptical defect are used: (a) When the major axis of the elliptical defect is along the $y$-direction, (b) When the major axis of the elliptical defect is along the $x$-direction.

The obtained results are illustrated in the Fig. 7b. When we compare obtained results in Fig. 7 with those in Fig. 6, the translation of the image shows improvements, the image is clearer.

Next, we focus on optimizing the geometric parameters of the ellipse in order to achieve a better lens resolution. We use the following design dimensions: major axis and minor axis lengths of $6 a$ and $3 a$, respectively. With this optimal configuration, we obtain a significant offset shift in the image position in the $x$-direction, corresponding to $2 a$.

In Fig. 8 transmission spectra (calculated by deploying the FDTD method) is shown for the PC superlens structure geometries illustrated in Fig. 7a,b.

Next, we further investigate and optimize the geometry of the ellipse in terms further improving the image resolutions. Figure 9 illustrates the image positions for two orientations of the ellipse as follows: The image of the position when the major axis is along $y$-direction and the small axis is along $x$-direction, whereas Fig. 10 illustrates the reversed case.

To further check the defect shape behaviour of the structure shown in Fig. 7, we calculate the transmission spectra and the obtained results are illustrated in Fig. 11. As can be seen from this figure, the normalised transmission peak of $90 \%$ is achieved at the normalised frequency $0.29 a / \lambda$ when the long axis of the elliptical defect is along the $y$-direction. However, for configuration when the long axis is along the $x$-direction, a slightly lower transmission of $80 \%$ is achieved at the same normalised frequency of $0.29 a / \lambda$.

To study the elliptical defect parameters and their influence on the vertical image shift, we slightly modify the orientation of the defect, whereas the shape is maintained the same. We have considered a set of numerical simulations of the light propagation for two values of the angle between the long axe of the elliptical defect and the $y$-axes as depicted in Figs 9 and 10. It is obvious from this that when the angle $\theta$ is equal to $30^{\circ}$ in the anticlockwise direction, the image is translated in vertical direction by a distance equal to $142 \mathrm{~nm}$ (the image is shift down). However, the image is shifted with the same value $(142 \mathrm{~nm})$ and for the same value of angle $\theta$ but in the clockwise direction, the image is shift upwards, as shown Fig. 10.

In Fig. 11. We illustrate the FDTD calculation of the normalised transmission response of the PC structure for the both value of the rotation angle $\theta$ that achieve a strong convergence of the lens, operating at the visible 


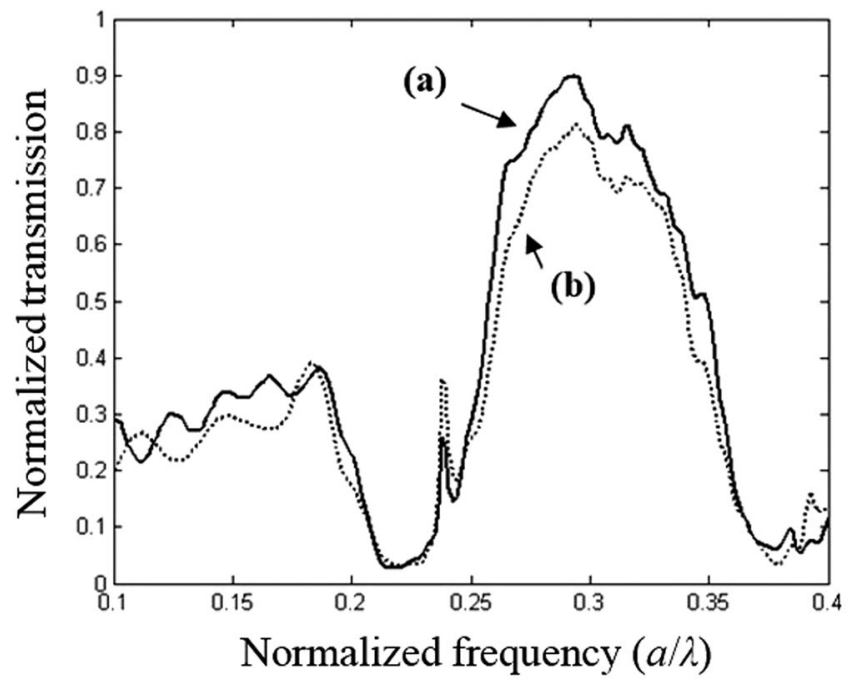

Figure 8. Time-domain simulation of the transmission spectra, when two different orientation of the elliptical defect are used: (a) when the major axis of the elliptical defect is along the $y$-direction, (b) when the major axis of the elliptical defect is along the $x$ direction, the image is strongly affected and shifted to a longer distance of about $\Delta x=284 \mathrm{~nm}$ in the $x$-direction.

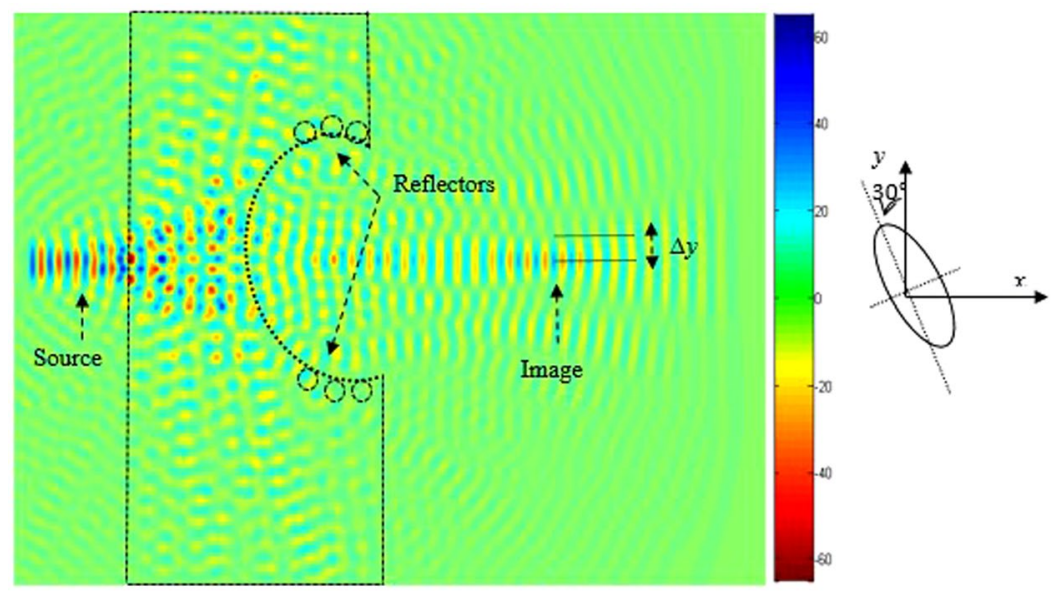

Figure 9. Time-domain simulation showing the imaging effect for a rotation angle $\theta=30^{\circ}$ of the elliptic defect in the anticlockwise direction. The image is shift down along $y$-direction with a distance $-142 \mathrm{~nm}$, and is clearly shown in its location.

frequencies. As shown in this figure, there is a shift of the image upwards with a distance of $\Delta y=142 \mathrm{~nm}$ (up) when the rotation angle $\theta$ is equal to $30^{\circ}$.

A shift in the downwards with a distance $\Delta y=-142 \mathrm{~nm}$ (dawn) is obtained when angle $\theta$ is equal to $30^{\circ}$ in the indirect sense. The spectra for $\Delta y=0$, is calculated when the angle $\theta$ is equal to zero. Moreover, if we compare these spectra, we note that the amplitude of the light is significantly higher from $0,25 a / \lambda$ to $0.35 \mathrm{a} / \lambda$, which shows that the field is coupling efficiently in the PC structure with defect due to negative refraction.

Next, we study the expansion of the source spot size on the images overlapping, in Fig. 12 we illustrate the snapshot of the electric field when the thickness $(t)$ of the source is set to $1.6 a$. It can be clearly seen that the formed images are confined into the plane and not coupled to each other. In case when we increase $t=3.6 a$, the snapshot of the images are less confined with intensities decreases dramatically compared to the source as shown in Fig. 13. The reason for the decreases of the image intensity is that the excited energy expands over wide range of photon energy resulting in less confinement which affects the image. It can be noticed that, the focal length is $2504.6 \mathrm{~nm}$ away from the concave face, which is smaller than the focal length (around $25 \mu \mathrm{m}$ ) reported in ref. ${ }^{41}$.

Next, in order to analyze and obtain higher quality resolution, we kept $t=1.6 a$, and we placed a second source (source 2) parallel to the source 1, as illustrated in Fig. 14. Source 1 and source 2 are separated by a distance $d$. We investigate the image intensity image interference at the focal length by optimizing the separation distance between two sources. In in Fig. 15, we have illustrated the light intensity when the separation distance between 


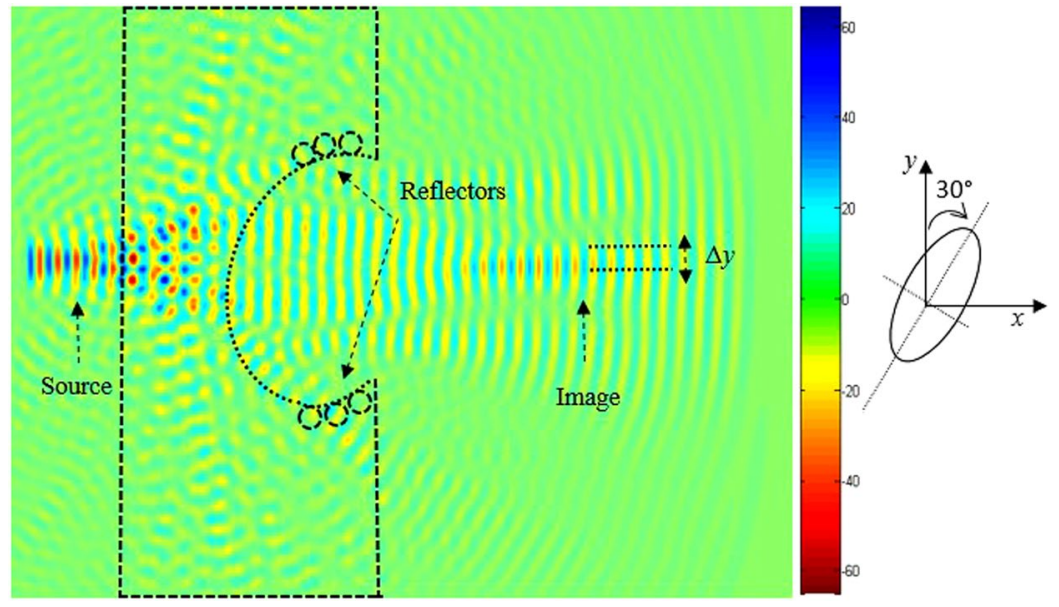

Figure 10. Time-domain simulation showing the imaging effect for a rotation angle $\theta=30^{\circ}$ of the elliptic defect in the clockwise direction. The image is shift upwards along $y$-direction with a distance of $142 \mathrm{~nm}$. A schematic of the elliptical defect orientation is also indicated.

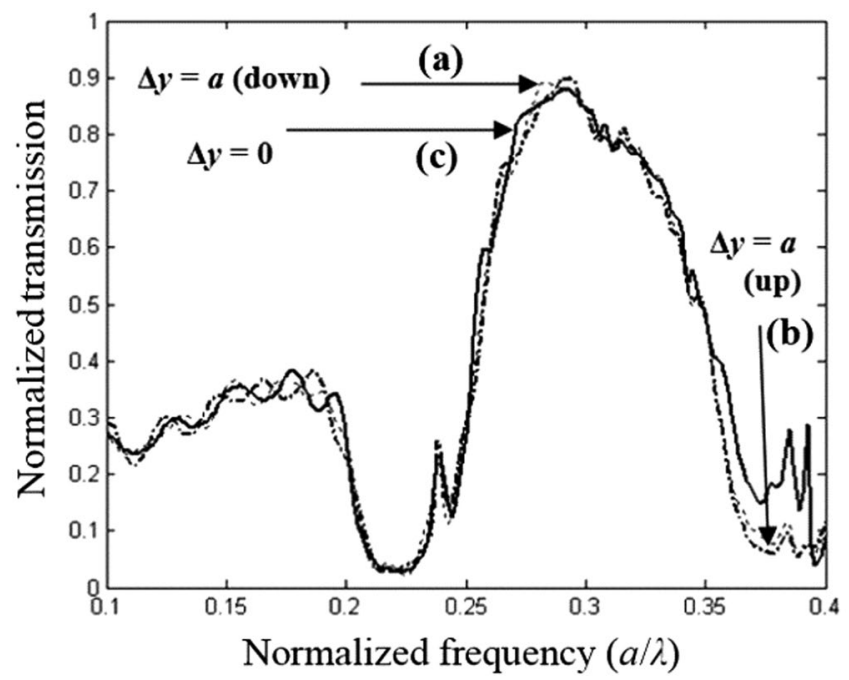

Figure 11. Time-domain simulation of the transmission spectrum, when two different orientation of the elliptical defect are used: (a) when the orientation angle of the elliptical defect is $\theta=30^{\circ}$ on the right, the image is strongly affected and shifted down to a longer distance of about $\Delta y=-142 \mathrm{~nm}$ along the $y$-direction, (b) when the orientation angle of the elliptical defect is $\theta=30^{\circ}$ on the left, the image is strongly affected and shifted up to a longer distance of about $\Delta y=142 \mathrm{~nm}$ along the $y$ direction, (c) when the major axis of the elliptical defect is along the $y$-direction $\left(\theta=0^{\circ}\right)$ which corresponds to the initial position of the image.

two sources is $d=0$. It is clear from our calculations shown in Fig. 15, that the images are not separated, they are overlying on each other.

Next, we further investigate and optimize the effect of distance $d$ on the image interferences. We increase the distance between sources $d$ to $0.55 \lambda$, as illustrated in Fig. 16. From this figure, it can be clearly seen that the image intensities are still interfering with each other. This shows that the sources are not separated enough, their spot sizes still interact preventing the separation.

We keep increasing (optimizing) $d$ which is set to $0.56 \lambda$. The image intensities along with the electrical field image snapshots are calculated and illustrated in Figs 17 and 18, respectively.

The FWHM comparison for the above structure geometries (illustrated in Figs 3, 4, 6a,b, 7a,b, 9, 10 and 14 are illustrated in Table 1. The highest FWHM is Fig. 2, due the fact that reflectors are not yet introduced, and also due to the existence of the two light sources. One can see that FWHM changes slightly in Figs 4, 6a,b, 7a,b, 9 and 10 , these values are close to each other due to the angle $\theta$ which displaces the image along $x$ and $y$ directions, therefore there is not so much effect on the light intensity.

From our calculations of the intensity at the plane image as a function of the position, illustrated in Fig. 18, it is clear that the formed images are separated and not coupled anymore, therefore the desired resolution is realized 


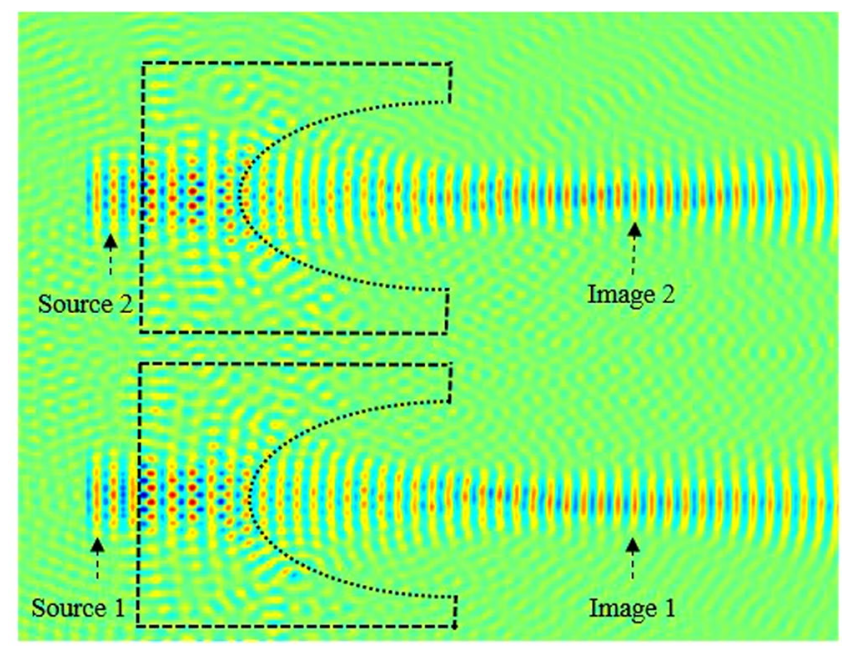

Figure 12. Snapshot of the electric field when the thickness $(t)$ of the source is set to $t=1.6 a$.

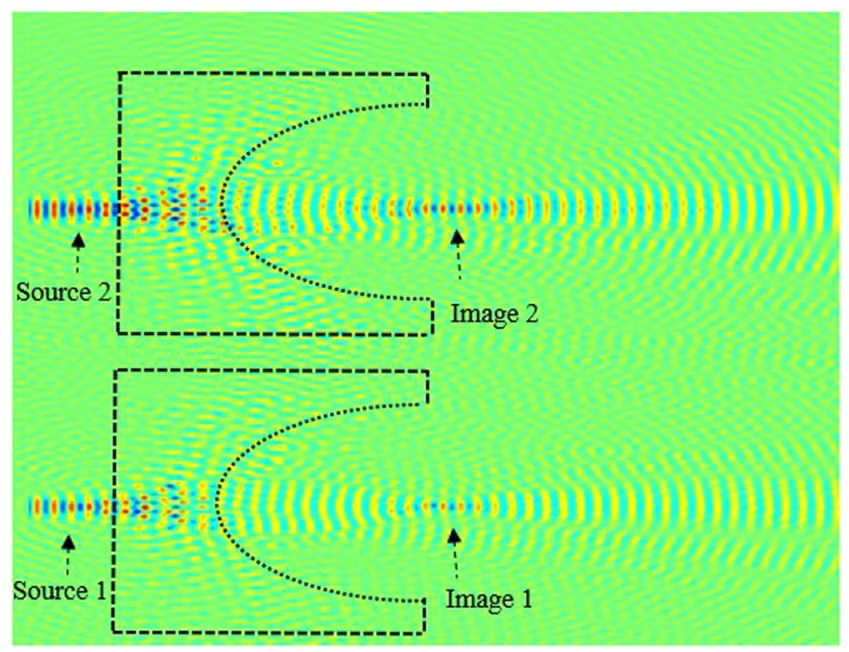

Figure 13. Snapshot of the electric field when the thickness $(t)$ of the source is set to $t=3.6 a$.

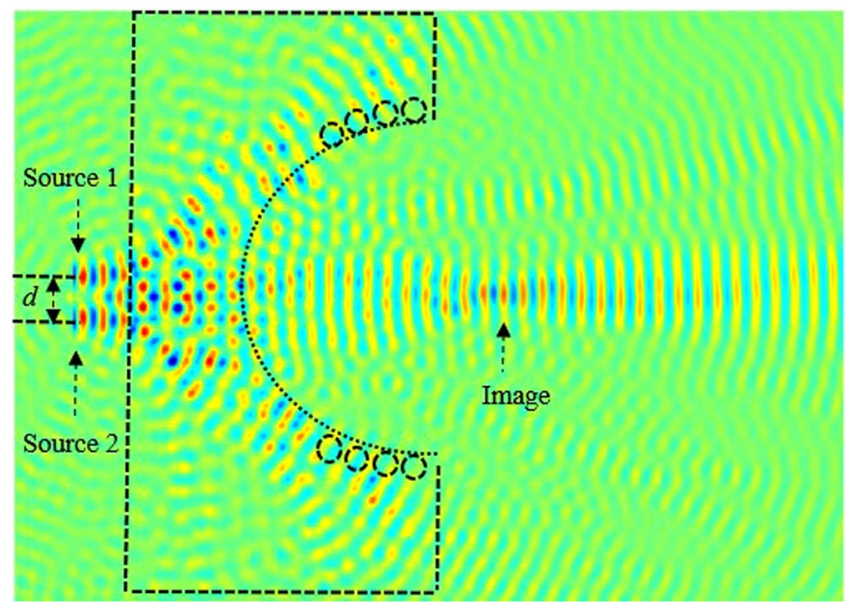

Figure 14. Schematics of the image when sources are separated by distance $d$. 


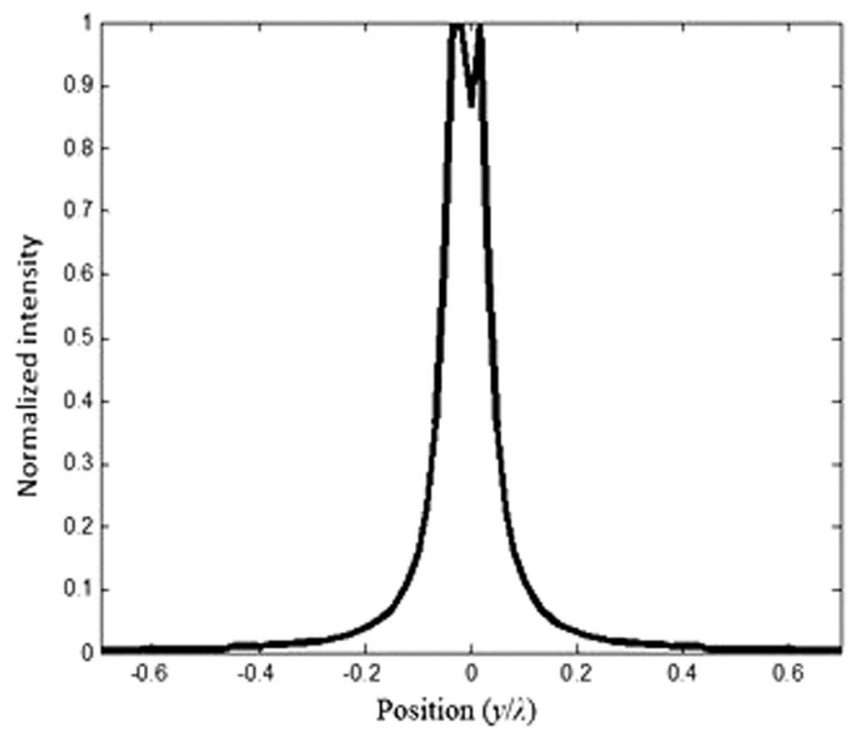

Figure 15. Illustration of the image interference when the separation distance $d=0$.

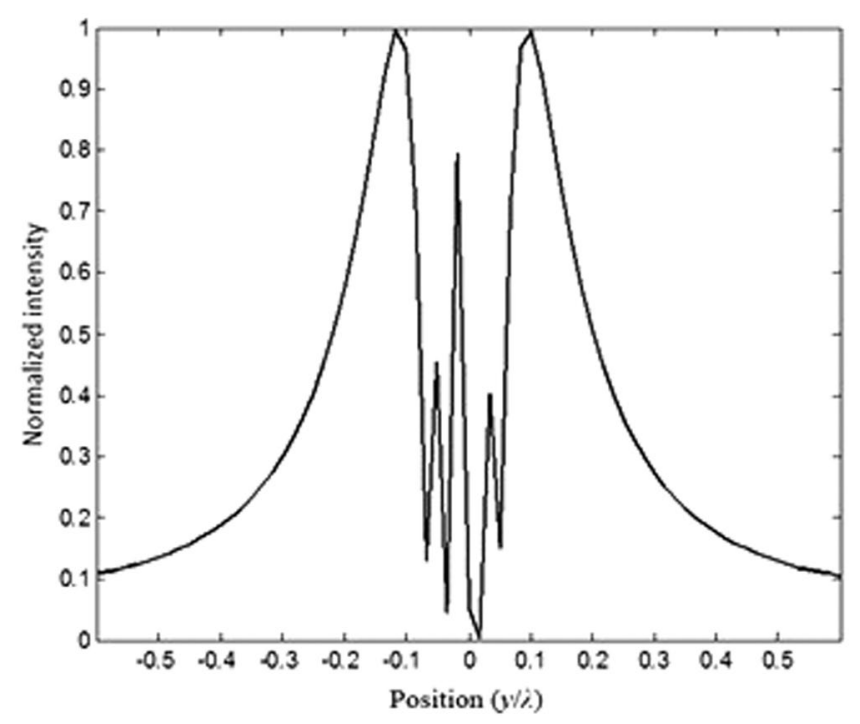

Figure 16. Illustration of the image interference when $d=0.55 \lambda$.

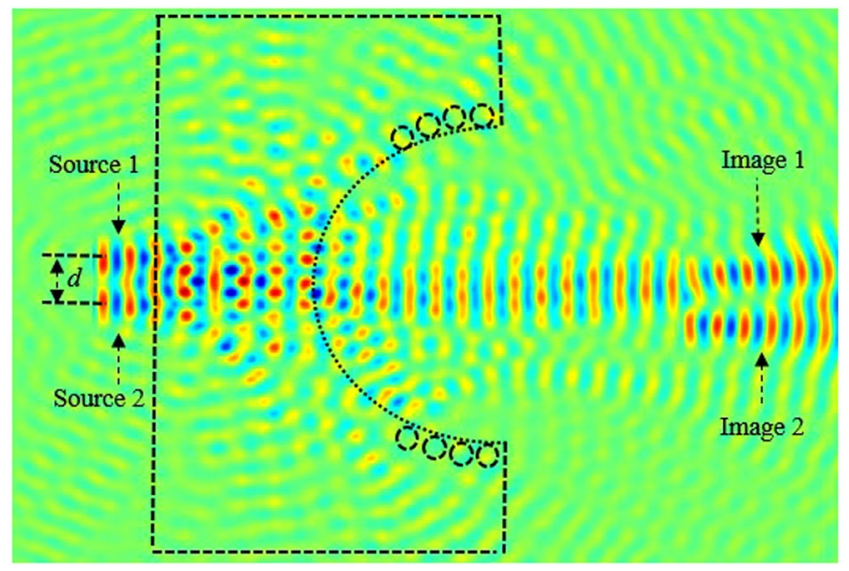

Figure 17. Snapshot of the electric field when the separation distance $d$ is set to $d=0.56 \lambda$ and $t=1.6 a$. 


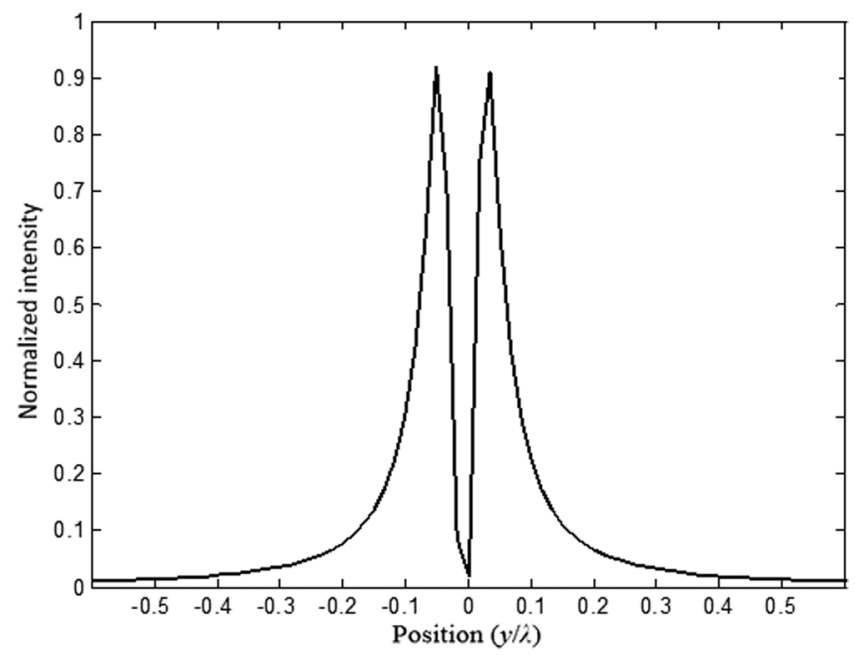

Figure 18. Illustration of the image interference when $d=0.56 \lambda$.

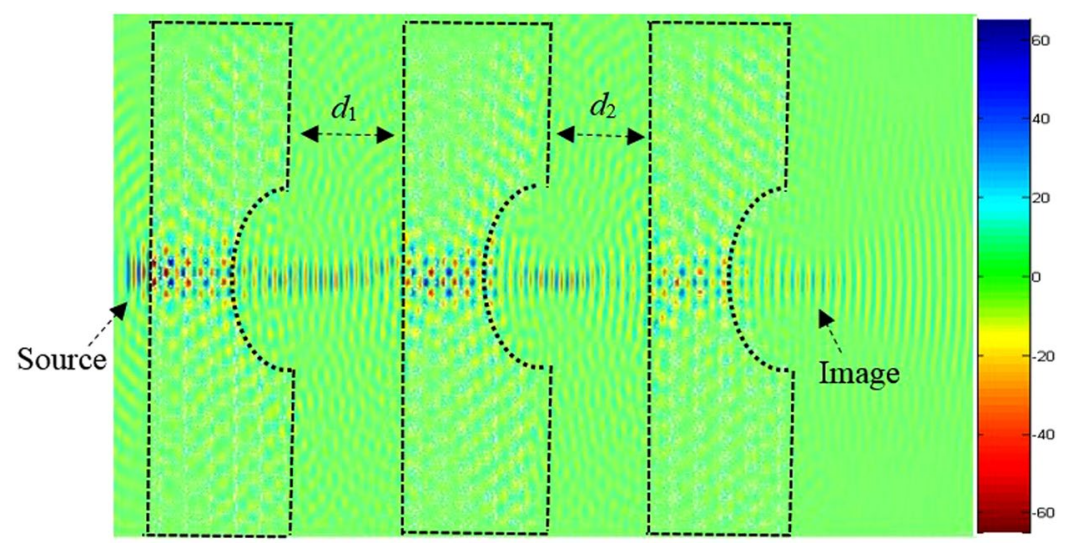

Figure 19. Snapshot of the electric field for three lenses when $d_{1}=d_{2}=188 \mathrm{~nm}$.

\begin{tabular}{|l|l|l|l|l|l|l|l|l|l|}
\hline Figure & 3 & 4 & $6 \mathrm{a}$ & $6 \mathrm{~b}$ & $7 \mathrm{a}$ & $7 \mathrm{~b}$ & 9 & 10 & 14 \\
\hline FWHM $(\mathrm{nm})$ & 1120 & 520 & 526 & 531 & 529 & 533 & 514 & 519 & 601 \\
\hline
\end{tabular}

Table 1. Obtained FWHM for different structure geometries.

by optimizing the thickness $(t)$ and the distance $d$ between the two sources. This can also be confirmed from the snapshots of the electric field shown in Fig. 16. It is clear from both Figs 17 and 18, that the images are no longer coupled.

In order to investigate the length of the arrayed structure, we consider three sources separated by $d_{1}$ and $d_{2}$. Initially, we kept fixed the following lens array design parameters; $d_{1}=d_{2}=f=1500 \mathrm{~nm}=3.1 \lambda$, and performed the evolution of the electric field along the array. The snapshot of the electric field evolution is illustrated in Fig. 19. It is evident form this figure, that the image is as clear as the source. Next, we have decreased the distance $d_{1}=d_{2}=f=750 \mathrm{~nm}=3.1 \lambda / 2$, the obtained image is has been affected, the image quality has been reduced. Subsequently, we further decreased the lens array parameters; $d_{1}=d_{2}=f=375 \mathrm{~nm}=3.1 \lambda / 4$, and $d_{1}=d_{2}=f=188 \mathrm{~nm}$. It is evident from our calculations that the image resolution quality is very low, in other words the quality of the image is reduced significantly when compared to obtained image when $d_{1}=d_{2}=f=1500 \mathrm{~nm}=3.1 \lambda$. Therefore based on our simulations, in order to obtain a high quality image resolution, the light sources should be kept at equidistance with the following parameters; $d_{1}=d_{2}=f=1500 \mathrm{~nm}$. In order to further analyse the image quality we have performed field intensity simulations for various distances between lenses, such as $d_{1}$ and $d_{2}$. Our study shows that when $d_{1}$ and $d_{2}$ are equal to $1500 \mathrm{~nm}$ its maximum is at about $16 \mathrm{a}$ a, the width of the field intensity is narrow, also the electric field propagates via longer distance where the losses are small, and therefore the image quality is higher. However, when the distances $d_{1}$ and $d_{2}$ decrease, the intensity decreases and its width becomes more broaden and the formed image is affected as shown in Fig. 19 when $d_{1}=d_{2}=188 \mathrm{~nm}$. 


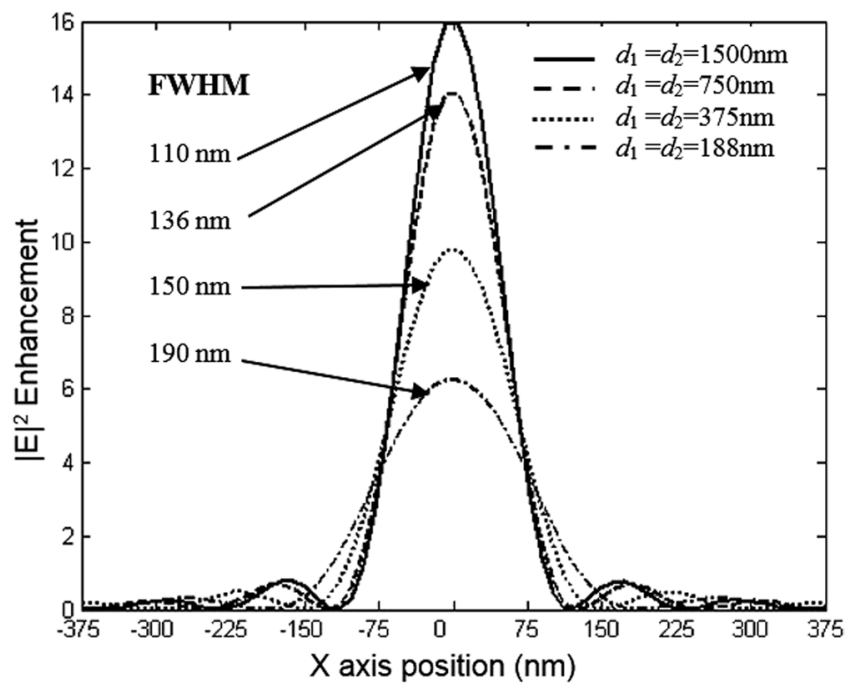

Figure 20. FWHM for various values of lens distances $d_{1}$ and $d_{2}$.

We have also calculated the light intensity $\mathrm{E}^{2}$ for different values of $d_{1}$ and $d_{2}$ where we determine the FWHM, as shown in Fig. 20. As can be seen from this figure, the light intensity $\mathrm{E}^{2}$ has been enhanced significantly when $d_{1}=d_{2}=1500 \mathrm{~nm}$

Based on the above proposed design, many other superlenses with desired image projection and resolution can be designed and integrated into a single platform ${ }^{27}$. Integration of the proposed superlenses into a single platform with precise accuracy will have significant effect on creating new M-Lens that will be consisted of a number of such superlens where each superlens could have different design constructions with precise required/ desired image projection. In this regard, the proposed superlens has significant advantages when capturing the pixels size of and object. The proposed superlens can be designed even as small as the pixel size. Current conventional microlens arrays used for imaging suffer from high light losses, low (poor) resolution, cannot determine the depth in third dimensions, they have limited angle of view. It is also known that the resolution of current optical devices is limited by the light operating wavelength. In particular, any current conventional lens cannot be fabricated and are unable to deal with images of the objects smaller than the operating wavelength. So, the sharpness of the image of the conventional lens is limited by the light operating wavelength. In order to achieve a strong coverage of the beam, several lenses should be integrated on the single chip, therefore the size of the device would be increased. Also when several lenses are integrated in a single platform the design of each lens should be optimized and adjusted in order to maintain the image resolution quality.

\section{Conclusion}

In this paper, we have demonstrated an ultra-compact imaging lens based metamaterial PC structure operating at visible wavelength. The proposed structure consists of a $\mathrm{GaP} / \mathrm{Ag} / \mathrm{GaP}$ metamaterial with a concave geometry shape with defects. We have used 2D FDTD numerical simulations to design and investigate a feasibility study of the proposed superlenses and integrate some of them in a single platform at operating visible wavelength. A comprehensive analysis of the geometric superlens and their integration parameters are performed. It is found that the lensing shift of the image in both directions, for each superlens, is strongly dependent on the angle and the shape of the introduced defect. The influence of the angle and shape of defects and the distance between integrated superlenses on the quality of the image resolution have been analyzed. Effects of the light coupling between integrated lenses on image quality resolution has been analyzed. Our initial feasibility model clearly demonstrates the proof-of-concept (POC) which verifies that the proposed idea have the potential to revolutionize the imaging technology for real-world light field imaging application. In this POC, we have demonstrated visible light control in 2D plane $(x$ and $y)$ imaging. Controlling and manipulation of the light in desired $3^{\text {rd }}$ dimension for each integrated lens, while maintaining high imaging resolution, is challenging, therefore our future study will be focused on $3 \mathrm{D}$ design and integration of the proposed superlenses, in a single platform, with high imaging resolution, operating at visible wavelength.

\section{References}

1. Abbe, E. Beiträgezurtheorie des mikroskops und der mikroskopischenwahrnehmung. Arch. Mikr. Anat. 9, 413-418, https://doi. org/10.1007/BF02956173 (1873).

2. Stephenson, J. W. Observations on professor Abbe's experiments illustrating his theory of microscopic vision. Am. Mon. Microsc. J. 17, 82-88, https://doi.org/10.1111/j.1365-2818.1877.tb04739.x (1877).

3. Zhang, X. \& Liu, Z. Superlenses to overcome the diffraction limit. Nature Materials 7, 435-441, https://doi.org/10.1038/nmat2141 (2008).

4. Rittweger, E., Young Han, K., Irvine, S. E., Eggeling, C. H. \& Hell, S. W. STED microscopy reveals crystal colourcentres with nanometric resolution. Nature Photonics 3, 14-147, https://doi.org/10.1038/nphoton.2009.2 (2009).

5. Vodo, P., Parimi, P. V., Lu, W. T. \& Sridhar, S. Focusing by planoconcavelens using negative refraction. Appl. Phys. Lett. 20, 201108, https://doi.org/10.1063/1.1927712 (2005).

6. Wood, B., Pendry, J. B. \& Tsai, D. P. Directed subwavelength imaging using a layered metal-dielectric system. Phys. Rev. B 74, 115116, https://doi.org/10.1103/PhysRevB.74.11511 (2006). 
7. Govyadinov, A. A. \& Podolskiy, V. A. Metamaterial photonic funnels for subdiffraction light compression and propagation. Phys. Rev. B 73, 155108, https://doi.org/10.1103/PhysRevB.73.155108 (2006).

8. Jacob, Z., Alekseyev, L. V. \& Narimanov, E. Optical Hyperlens: Far-field imaging beyond the diffraction limit. Optics Express 14, 8247-8256, https://doi.org/10.1364/OE.14.008247 (2006).

9. Zhaowei, L., Hyesog, L., Yi, X., Cheng, S. \& Xiang, Z. Far-Field Optical Hyperlens Magnifying Sub-Diffraction-Limited Objects. Science 315, 1699-1701, https://doi.org/10.1126/science.1137368 (2007).

10. Khorasaninejad, M. et al. Metalenses at visible wavelengths: Diffraction-limited focusing and subwavelength resolution imaging. Science 352, 6290, https://doi.org/10.1126/science.aaf6644 (2016).

11. Yue, L., Yan, B. \& Wang, Z. Photonic nanojet of cylindrical metalens assembled by hexagonally arranged nanofibers for breaking the diffraction limit. Optics Letters 7, 1336-1339, https://doi.org/10.1364/OL.41.001336 (2016).

12. Sharkawy, A. et al. Modulating dispersion properties of low index photonic crystal structures using microfluidics. Optics Express 13(8), 2814-2827, https://doi.org/10.1364/OPEX.13.002814 (2005).

13. Jennefir, L. D. et al. Tight control of light beams in photonic crystals with spatially-variant lattice orientation. Optics Express 22(21), 25788-25804, https://doi.org/10.1364/OE.22.025788 (2014).

14. Li, Y. H., Fu, Y. Q., Minin, O. V. \& Minin, I. V. Ultra-sharp nanofocusing of graded index photonic crystal-based lenses perforated with optimized single defect. Optical Materials Express 6(8), 2628-2636, https://doi.org/10.1364/OME.6.002628 (2016).

15. Lin, C. et al. Efficient terahertz coupling lens based on planar photonic crystals on silicon on insulator. Optics Letters 30(11), 1330-1332, https://doi.org/10.1364/OL.30.001330 (2005).

16. Lu, Z. et al. Photonic Crystal Planar Lens Working at Low Frequencies. Proceedings of SPIE - The International Society for Optical Engineering 5733-444-449, https://doi.org/10.1117/12.589299 (2005).

17. Naserpour, M., Carlos, J., Zapata-Rodríguez, J. C. \& Mahdieh, H. Plano-concave microlenses with epsilon-near-zero surface-relief coatings for efficient shaping of nonparaxial optical beams. Optics \& Laser Technology 98(1), 152-157, https://doi.org/10.1016/j. optlastec.2017.07.053 (2018).

18. Naserpour, M., Zapata-Rodríguez, J. C., Díaz-Aviñó, C. \& Mahdieh, H. Metacoatings for wavelength-scale, high-numerical-aperture plano-concave focusing lenses. Journal of the Optical Society of America B 33(10), 2120-2128, https://doi.org/10.1364/ JOSAB.33.002120 (2016).

19. Minin, I. V., Minin, O. V., Triandaphilov, Y. R. \& Kotlyar, V. V. Focusing Properties of Two Types of Diffractive Photonic Crystal Lens. Optical Memory and Neural Networks 17(3), 244-248, https://doi.org/10.3103/S1060992X08030089 (2008).

20. Prasad, T., Colvin, V. L. \& Mittleman, D. M. The effect of structural disorder on guided resonances in photonic crystal slabs studied with terahertz time-domain spectroscopy. Optics Express 15(25), 16954-16965, https://doi.org/10.1364/OE.15.016954 (2007).

21. AbdelMalek, F., Belhadj, W., Haxha, S. \& Bouchriha, H. Realization of High Coupling Efficiency by Employing Concave Lens Based on 2D photonic Crystals with Negative Refractive Index. Journal of Lightwave Technol. 25(10), 3168-3174, https://doi.org/10.1109/ JLT.2007.904027 (2007).

22. Lippmann, G. La Photographie Integrale. Comtes Rendus. Academie des Sciences 146, 446-451 ISBN-10: 2800147954 (1908).

23. Hahne, C., Aggoun, A., Velisavljevic, V., Fiebig, S. \& Pesch, M. Triangulation Geometry in a Standard Plenoptic Camera. International Journal of Computer Vision 126(1), 21-35, https://doi.org/10.1007/s11263-017-1036-4 (2018).

24. Řeřabek, M. \& Ebrahimi, T. New Light Field Image Dataset. 8th International Conference on Quality of Multimedia Experience (QoMEX), Lisbon, Portugal, https://infoscience.epfl.ch/record/218363/files/Qomex2016_shortpaper.pdf (2016).

25. Hane, C., Aggoun, A., Haxha, S., Vladan \& Fernández, V. J. C. J. Light field geometry of a standard plenoptic camera. Optics Express 22(22), 26659-26673, https://doi.org/10.1364/OE.22.026659 (2014).

26. Aggoun, A. 3D Holoscopic ImagingTechnology for Real-Time Volume Processing and Display', High-Quality Visual Experience Signals and Communication Technology, Book Chapter 18, ISBN 978-3-642-12802-8, IV, 411-428 (2010).

27. Haxha, S., AbdelMalek, F. \& Aggoun, A. International Patent Application No. PCT/GB2015/051310: United Kingdom Application No. $1407922.2(2014)$

28. Guo, Y., Bennamoun, M., Sohel, F., Lu, M. \& Wan, J. 3D object recognition in cluttered scenes with local surface features: A survey. Pattern Analysis and Machine Intelligence. IEEE Transactionson 11, 2270-2287, https://doi.org/10.1109/TPAMI.2014.2316828 (2014).

29. Zhao, W. L. \& Ngo, C. W. Flip-invariant SIFT for copy and object detection. Image Processing, IEEE Transactions on 3, 980-991, https://doi.org/10.1109/TIP.2012.2226043 (2013).

30. Zhao, X., Zhang, C., Xu, L., Yang, B. \& Feng, Z. IGA-based point cloud fitting using B-spline surfaces for reverse engineering. Information Sciences 245, 276-289, https://doi.org/10.1016/j.ins.2013.04.022 (2013).

31. Matchmoving, T. D. The Invisible Art of Camera Tracking. 2nd Edition. John Wiley \&Sons, ISBN: 978-1-118-35205-2 (2012).

32. Ouerghi, F. et al. Nanophotonic Sensor Based on Photonic Crystal Structure Using Negative Refraction for Effective Light Coupling. IEEE Journal of Lightwave Technology 15, 3269-3274, https://doi.org/10.1109/JLT.2009.2021488 (2009).

33. Taflove, A. \& Hagness, S. C. Computational Electrodynamics: The Finite-Difference Time-Domain Method. 2nd ed., Norwood, MA: Artech House, ISBN: 9781580538329 (2000).

34. Yee, K. S. Numerical solution of initial boundary value problems involving Maxwell's equations in isotropic media. IEEE Trans. Antennas Propagat. AP-14(3), 302-307, https://doi.org/10.1109/TAP.1966.1138693 (1966).

35. B' 'erenger, J. P. A Perfect Matched Layer for the Absorption of Electromagnetic Waves. J. Comput. Phys. 114, 185-200, https://doi. org/10.1006/jcph.1994.1159 (1994).

36. Haxha, S., Belhadj, W., AbdelMalek, F. \& Bouchriha, H. Analysis of Wavelength Demultiplexer based on Photonic Crystals. IEE Proceedings - Optoelectronics, 152(4), 193-198, https://doi.org/10.1049/ip-opt:20050003 (2005).

37. Haxha, S. \& AbdelMalek, F. A Novel Design of Photonic Crystal Lens Based on Negative Refractive Index Progress in Electromagnetics Research Symposium (PIERS). The 23rd PIERS 2008 in Hangzhou, China - Invited paper, Published: March (2008).

38. Lewis, P. \& Lee, P. Band Structure and Electronic Properties of Silver. Phys. Rev. 175, 795, https://doi.org/10.1103/PhysRev.175.795 (1968).

39. Christensen, N. E. The Band Structure of Silver and Optical Interband Transitions. Phys. Status Solidi B 54, 551-563, https://doi. org/10.1002/pssb.2220540219 (1972).

40. Nieto-Vesperinas, M. Problem of image superresolution with a negative-refractive-index slab. J. Opt. Soc. Am. A 21, 491-498, https://doi.org/10.1364/JOSAA.21.000491 (2004).

41. Menona, L. et al. Negative index metamaterials based on metal-dielectric nanocomposites for imaging applications. Appl. Phys. Lett. 93, 123117, https://doi.org/10.1063/1.2945647 (2008).

\section{Author Contributions}

$\mathrm{S}$. Haxha proposed the idea and the structures, performed image projections in $x$ - and $y$-coordinates, contributed in the numerical modelling and coordinated and led the writing of the proposed work. F. AbdelMalek contributed on the numerical modelling of the structures configuration jointly with F. Ouerghi. F. Ouerghi also contributed on simulation of the transmission spectrum. M.D.B. Charltonperformed the details of the metamaterial lens fabrication. A. Aggoun contributed on the 2D and 3D images and image projections. X. Fang contributed on the expansion of the source spot size on the images overlapping and coupling of the light when lenses are integrated in the single platform. 


\section{Additional Information}

Competing Interests: The authors declare no competing interests.

Publisher's note: Springer Nature remains neutral with regard to jurisdictional claims in published maps and institutional affiliations.

(c) (i) Open Access This article is licensed under a Creative Commons Attribution 4.0 International License, which permits use, sharing, adaptation, distribution and reproduction in any medium or format, as long as you give appropriate credit to the original author(s) and the source, provide a link to the Creative Commons license, and indicate if changes were made. The images or other third party material in this article are included in the article's Creative Commons license, unless indicated otherwise in a credit line to the material. If material is not included in the article's Creative Commons license and your intended use is not permitted by statutory regulation or exceeds the permitted use, you will need to obtain permission directly from the copyright holder. To view a copy of this license, visit http://creativecommons.org/licenses/by/4.0/.

(c) The Author(s) 2018 